\title{
Mathematical modeling and predicting the current trends of human population growth in Bangladesh
}

\author{
Hironmoy Mondol*, Uzzwal Kumar Mallick, Md. Haider Ali Biswas \\ Mathematics Discipline, Khulna University, Khulna 9208, Bangladesh
}

Corresponding Author Email: hironmoyku@gmail.com

https://doi.org/10.18280/mmc_d.390101

Received: 10 March 2018

Accepted: 15 June 2018

\section{Keywords: \\ exponential growth model, logistic population model, carrying capacity, population growth, vital coefficient}

\begin{abstract}
Bangladesh is an overpopulated and the most densely populated country. It is the world's eighth-most populous country in south Asia with over 160 million people. Population problem in Bangladesh is one of the most burning issues in the recent years. So the increasing trend in population is a great threat to the nation and for this reason, the projection of the population of Bangladesh is essential. The purpose of this paper is to model and design the population growth in Bangladesh to predict the future population size. The exponential and the logistic growth models are applied to predict the population of Bangladesh during 1980 to 2080 using the actual data from 1980 to 2016 . By using the exponential growth model, the predicted growth rate has been estimated approximately $2.67 \%$ and the population of Bangladesh has been predicted to be 1191 million in 2080 We have determined the carrying capacity $(K)$ and vital coefficients $a$ and $b$ for the population prediction in vein of logistic growth model. Thus, the population growth rate of Bangladesh according to the logistic model has been estimated approximately $4.03 \%$ and the total population of Bangladesh has been predicted to be 245 million in 2080 .
\end{abstract}

\section{INTRODUCTION}

Population is the most vital element of world but population projection has become one of the most serious problems in the world. Population sizes and growth in a country directly influence the situation of the economy, policy, culture, education and environment of that country and determine exploring the cost of natural resources [24]. Every government and collective sectors always require proper idea about the future size of various subsistence like population, resources, demands and consumptions for their future activities [11, 23]. To obtain this information, the behavior of the connected variables is analyzed based on the previous data by the statisticians and mathematicians and using the conclusions drawn from the analysis, they make future projections of the aimed at variable $[2,11,4]$. There are enormous concerns about the consequences of human population growth for social, environmental and economic development which Intensify all these problems in population growth. Mathematical modeling is a broad interdisciplinary science that uses mathematical and computational techniques to model and elucidate the phenomena arising in real life problems [25]. Thus, it is a process of mimicking reality by using the language of mathematics in terms of differential equations which describe the changing phenomena of the underlying systems. The population models determine the present state in terms of the past and the future state in terms of its present state which describes a typical human way of coping with the reality. The main reason for solving many differential equations is to learn the behavior about an underlying physical process that the equation is believed to model $[3,5]$. Many people examine population growth through observation, experimentation or through mathematical modeling. The population models are used in forecasting of population growth, population decaying, maximum or minimum production, food preserving, environmental, capacity and many other applications [1]. Mathematical models can take many forms including dynamical systems, statistical models and differential equations [12]. These and other types of models can overlap, with a given model involving a variety of abstract structures. A population model is a type of mathematical model that is applied to the study of population dynamics. Models allow a better understanding of how complex interactions and processes work [6]. Modeling of dynamic interactions in nature can provide a manageable way of understanding how numbers change over time or in relation to each other. We refer readers to $[7,19-21]$ for some recent studies on population models in analyzing biological systems.

In this paper, we discuss an overview of population growth models in terms of nonlinear differential equations in the form of mathematical modeling which have been applied to study the future prediction of human population in Bangladesh. The first order differential equations have been used to govern the growth of the human species. Two simple deterministic population models, namely, Malthusian growth model and logistic growth model have been studied and analyzed to discuss the dynamical behavior of the population viability analysis for both short-term and long-term prediction in Bangladesh. Our study shows that Malthusian model is more accurate for short-term prediction, but for long-term prediction, population growth is unrealistic. On the other hand, logistic model is more realistic than Malthusian model for long term 
prediction. Both the exponential and the logistic growth models have been applied to predict the population of Bangladesh during 1980 to 2080 using the actual data from 1980 to 2016 collected from the World Bank [23]. We have shown by the exponential growth model that the predicted growth rate is approximately $2.67 \%$ and the population of Bangladesh has been predicted to be 1191 million by 2080. In similar fashion, we have determined the carrying capacity $(K)$ and vital coefficients $a$ and $b$ for the population prediction in vein of logistic growth model. Our analysis shows that the population growth rate of Bangladesh according to the logistic model has been estimated approximately $4.03 \%$ and the total population of Bangladesh has been predicted to be 245 million in 2080. Our results show that the predicted populations of Bangladesh are very similar to the present trends of the population size.

\section{METHODOLOGY}

'Methodology' is more effective than the simple methods in which one intends to use for collecting data. It is often necessary to include a consideration of the concepts and theories which underlies the methods. For instance, if one intends to highlight a specific feature of a mathematical theory or test an algorithm for some aspects of information or test the validity of a particular system, we have to show that we understand the underlying concepts of the methodology.

\subsection{Malthusian model}

A Malthusian growth model is an essentially exponential growth based on a constant rate. The model is named after Thomas Robert Malthus. The Malthusian growth model is also known as a simple exponential growth model [7]. Malthusian model is single species population model. Let $t$ denotes the time and $y(t)$ denotes the number of individuals presents at a time $t$.

In practice $y(t)$ is a non-negative integer. We assume that $y(t)$ continuously differentiable [1]. The growth rate of population is the rate of at which population changes [5]. If the population $y(t)$ at time $t$ changes to $y(t+\delta t)$ the average per capital growth rate at the time $\delta t$ is $\frac{y(t+\delta t)-y(t)}{y(t) \delta t}$.

Taking limit $\delta t \rightarrow 0$, we get the instantaneous growth rate at the time $\delta t$

$$
\lim _{\delta t \rightarrow 0} \frac{y(t+\delta t)-y(t)}{y(t) \delta t}=\frac{y^{\prime}(t)}{y(t)}
$$

Now let,

$b=$ Intrinsic birth rate.

$=$ The average number of off spring born per individuals per time.

$d=$ Intrinsic death rate.

$=$ The fraction of individuals of the population dies per unit time.

$r=b-d$

$=$ Intrinsic growth rate of the population.

$=$ Excess of birth over death per unit time individuals.

Now, we consider a single species of population, the growth model is described by

$$
\begin{aligned}
& \frac{y^{\prime}(t)}{y(t)}=r \\
& \quad \Rightarrow y^{\prime}(t)=r y(t)
\end{aligned}
$$

with the initial population, $y\left(t_{0}\right)=y_{0}>0$. Thus we have the mathematical model described the growth of single species population as

$$
y^{\prime}(t)=r y(t), \quad y\left(t_{0}\right)=y_{0}>0
$$

General solution of this equation (1) is $y(t)=c e^{r t}$. For the initial condition $y\left(t_{0}\right)=y_{0}$.

We have,

$$
\begin{aligned}
& y\left(t_{0}\right)=c e^{r t_{0}}=y_{0} \\
& \Rightarrow c=y_{0} e^{-r t_{0}} \\
& \therefore y(t)=y_{0} e^{-r t_{0}} e^{r t}=y_{0} e^{r\left(t-t_{0}\right)} \\
& \quad \text { Therefore, } \\
& y(t)=y_{0} e^{r\left(t-t_{0}\right)}
\end{aligned}
$$

which is known as Malthusian law of growth [5].

\subsection{Logistic model}

Logistic model is a modification of Malthusian model. The Logistic model is a model of population growth first published by a Belgian mathematical biologist Pierre Verhulst [6]. He observed that the population growth not only depends on the population size but also on how far this size is from its carrying capacity [10]. He modified Malthusian model to make a population size proportional to both the previous population and a new term [13].

$$
\frac{a-b p(t)}{a}
$$

where $a$ and $b$ are the vital coefficients of the population.

Now as the population value gets closer to $\frac{a}{b}$ this new term will become very small and tend to zero, which gives the right feedback to limit the population growth [15]. Thus the second term models the competition for available resources, which tends to unite the population growth [18]. So the modified equation using this new term is

$\frac{d p}{d t}=\frac{a p(t)[a-b p(t)]}{a}$

This equation is known as the logistic law of population growth model.

Solving (4) and applying the initial condition $p\left(t_{0}\right)=p_{0}$ then (4) become 
$\frac{d p}{d t}=a p-b p^{2}$

$\therefore \frac{d p}{p(a-b p)}=d t$

By the application of separation of variables

$\frac{1}{a}\left(\frac{1}{p}+\frac{b}{a-b p}\right) d p=d t$

Now integrating (6), we obtain

$\Rightarrow \frac{1}{a}[\ln p-\ln (a-b p)]=t+c$

At $t=0$, we get $p\left(t_{0}\right)=p_{0}$

$\therefore c=\frac{1}{a}\left[\ln p_{0}-\ln \left(a-b p_{0}\right)\right]$

Equation

$\frac{1}{a}[\ln p-\ln (a-b p)]=t+\frac{1}{a}\left[\ln p_{0}-\ln \left(a-b p_{0}\right)\right]$

becomes

$$
\therefore p=\frac{\frac{a}{b}}{1+\left(\frac{\frac{a}{b}}{p_{0}}-1\right) e^{-a t}}
$$

If we take the limit of equation (9) as $t \rightarrow \infty$, we get

$p_{\max }=\lim _{t \rightarrow \infty} p=\frac{a}{b}$

Taking $t=0, t=1$ and $t=2$ the values of $p_{0}, p_{1}$ and $p_{2}$ respectively then we get equation (9)

$$
\left.\begin{array}{l}
\frac{b}{a}\left(1-e^{-a}\right)=\frac{1}{p_{1}}-\frac{e^{-a}}{p_{0}} ; \\
\frac{b}{a}\left(1-e^{-2 a}\right)=\frac{1}{p_{2}}-\frac{e^{-2 a}}{p_{0}}
\end{array}\right\}
$$

Eliminating $\frac{b}{a}$, we have

$\Rightarrow e^{-a}=\frac{p_{0}\left(p_{2}-p_{1}\right)}{p_{2}\left(p_{1}-p_{0}\right)}$

Putting this value into the first equation of (11), we get

$$
\frac{b}{a}=\frac{p_{1}^{2}-p_{0} p_{2}}{p_{1}\left(p_{0} p_{1}-2 p_{0} p_{2}+p_{1} p_{2}\right)}
$$

Thus the limiting value of $p$ is given by

$$
p_{\max }=\lim _{t \rightarrow \infty} p=\frac{a}{b}=\frac{p_{1}\left(p_{0} p_{1}-2 p_{0} p_{2}+p_{1} p_{2}\right)}{p_{1}^{2}-p_{0} p_{2}}
$$

\section{RESULTS AND DISCUSSIONS}

To predict the future population of Bangladesh, we need to determine growth rate of Bangladesh using the exponential growth model in (2).

Using the actual population of Bangladesh in Table 1 below with $t=0$ i.e. $t_{0}$ corresponding to the year 1980, we have $P(0)=82.49$ i.e. $P_{0}=82.49$. We can solve for the growth rate $k$ the fact that $P(5)=94.28$ i.e. $P_{5}=94.28$ when $t=5$ i.e. $t_{5}$ then we get from the equation (2),

$\therefore P_{5}=P_{0} e^{k\left(\mathrm{t}_{5}-t_{0}\right)}$

$\Rightarrow 94.28=82.49 e^{k(5-0)}$

$\therefore k=0.03$.

Hence, the general solution is

$P(t)=82.49 e^{0.0267 t}$

This suggests the prediction rate of population growth is $2.67 \%$ in Bangladesh with the exponential growth model. With this we project the population of Bangladesh from 1980 to 2080. Again based on Table 1 , let $t=0,1$ and 2 correspond to the years 1980, 1981 and 1982 respectively. Then $P_{0}, P_{1}$ and $P_{2}$ also correspond $82.49,84.76$ and 87.06 (in million). Substituting the value of $P_{0}, P_{1}$ and $P_{2}$ into (14), we get

$P_{\max }=\frac{a}{b}=254.89 \times 10^{6}$

This is the predicted carrying capacity of the population of Bangladesh. From equation (12), we obtain $e^{-a}=0.96$ hence $a=-\ln (0.96)$. Therefore the value of $a=0.04$. This also implies that the predicted rate of population growth of Bangladesh is approximately $4 \%$ with the logistic growth model [13]. Now from equation (16), we obtain $b=1.58 \times 10^{-10}$. Substituting the values of $P_{0}, a$ and $b$ into equation (9) then we get

$P(t)=\frac{254893900}{1+2.08(0.96)^{t}}$ 
This equation is used to compute the predicted values of the population [17]. The predicted population of Bangladesh using both models is presented in the Table 1 from 1980 to 2016 with actual data [23].

Table 1. Projected population in Bangladesh from 1980 to 2080 using Exponential and Logistic growth models with help of actual data

\begin{tabular}{|c|c|c|c|c|c|}
\hline \multirow[t]{2}{*}{ Year } & \multirow{2}{*}{$\begin{array}{l}\text { Actual population } \\
\quad \text { (in millions) }\end{array}$} & \multicolumn{4}{|c|}{ Projected population (in millions) } \\
\hline & & Exponential model & $\begin{array}{l}\text { Absolute } \\
\text { Percentage } \\
\text { Error }\end{array}$ & Logistic model & $\begin{array}{l}\text { Absolute } \\
\text { Percentage } \\
\text { Error }\end{array}$ \\
\hline 1980 & 82 & 82 & $0.00 \%$ & 82 & $0.0006 \%$ \\
\hline 1981 & 84 & 84 & $0.03 \%$ & 84 & $0.002 \%$ \\
\hline 1982 & 87 & 87 & $0.04 \%$ & 87 & $0.003 \%$ \\
\hline 1983 & 89 & 89 & $0.02 \%$ & 89 & $0.01 \%$ \\
\hline 1984 & 91 & 91 & $0.01 \%$ & 91 & $0.07 \%$ \\
\hline 1985 & 94 & 94 & $0.01 \%$ & 94 & $0.18 \%$ \\
\hline 1986 & 96 & 96 & $0.02 \%$ & 96 & $0.34 \%$ \\
\hline 1987 & 99 & 99 & $0.02 \%$ & 98 & $0.53 \%$ \\
\hline 1988 & 102 & 102 & $0.01 \%$ & 101 & $0.71 \%$ \\
\hline 1989 & 104 & 104 & $0.12 \%$ & 103 & $0.86 \%$ \\
\hline 1990 & 107 & 107 & $0.33 \%$ & 106 & $0.95 \%$ \\
\hline 1991 & 109 & 110 & $0.66 \%$ & 108 & $0.97 \%$ \\
\hline 1992 & 112 & 113 & $1.08 \%$ & 111 & $0.93 \%$ \\
\hline 1993 & 114 & 116 & $1.59 \%$ & 113 & $0.85 \%$ \\
\hline 1994 & 117 & 119 & $2.14 \%$ & 116 & $0.77 \%$ \\
\hline 1995 & 119 & 123 & $2.72 \%$ & 119 & $0.71 \%$ \\
\hline 1996 & 122 & 126 & $3.32 \%$ & 121 & $0.67 \%$ \\
\hline 1997 & 124 & 129 & $3.95 \%$ & 124 & $0.64 \%$ \\
\hline 1998 & 127 & 133 & $4.64 \%$ & 126 & $0.60 \%$ \\
\hline 1999 & 129 & 137 & $5.42 \%$ & 129 & $0.53 \%$ \\
\hline 2000 & 132 & 140 & $6.29 \%$ & 131 & $0.40 \%$ \\
\hline 2001 & 134 & 144 & $7.27 \%$ & 134 & $0.24 \%$ \\
\hline 2002 & 137 & 148 & $8.34 \%$ & 136 & $0.03 \%$ \\
\hline 2003 & 139 & 152 & $9.53 \%$ & 139 & $0.23 \%$ \\
\hline 2004 & 141 & 156 & $10.86 \%$ & 142 & $0.57 \%$ \\
\hline 2005 & 143 & 160 & $12.35 \%$ & 144 & $1.00 \%$ \\
\hline 2006 & 144 & 165 & $14.01 \%$ & 147 & $1.53 \%$ \\
\hline 2007 & 146 & 169 & $15.82 \%$ & 149 & $2.14 \%$ \\
\hline 2008 & 147 & 174 & $17.74 \%$ & 152 & $2.77 \%$ \\
\hline 2009 & 149 & 178 & $19.69 \%$ & 154 & $3.36 \%$ \\
\hline 2010 & 151 & 183 & $21.61 \%$ & 156 & $3.87 \%$ \\
\hline 2011 & 152 & 188 & $23.48 \%$ & 159 & $4.27 \%$ \\
\hline 2012 & 154 & 193 & $25.32 \%$ & 161 & $4.58 \%$ \\
\hline 2013 & 156 & 199 & $27.15 \%$ & 164 & $4.83 \%$ \\
\hline 2014 & 159 & 204 & $28.29 \%$ & 166 & $4.45 \%$ \\
\hline 2015 & 161 & 210 & $30.29 \%$ & 168 & $4.72 \%$ \\
\hline 2016 & 162 & 215 & $32.38 \%$ & 171 & $4.99 \%$ \\
\hline 2017 & & 221 & & 173 & \\
\hline 2018 & & 227 & & 175 & \\
\hline 2019 & & 233 & & 177 & \\
\hline 2020 & & 240 & & 179 & \\
\hline 2021 & & 246 & & 182 & \\
\hline 2022 & & 253 & & 184 & \\
\hline 2023 & & 260 & & 186 & \\
\hline 2024 & & 267 & & 188 & \\
\hline 2025 & & 274 & & 190 & \\
\hline 2026 & & 281 & & 192 & \\
\hline 2027 & & 289 & & 193 & \\
\hline 2028 & & 297 & & 195 & \\
\hline 2029 & & 305 & & 197 & \\
\hline 2030 & & 313 & & 199 & \\
\hline 2031 & & 321 & & 201 & \\
\hline 2032 & & 330 & & 202 & \\
\hline 2033 & & 339 & & 204 & \\
\hline 2034 & & 348 & & 206 & \\
\hline 2035 & & 354 & & 207 & \\
\hline 2036 & & 367 & & 209 & \\
\hline 2037 & & 377 & & 210 & \\
\hline
\end{tabular}




\begin{tabular}{|c|c|c|c|c|c|}
\hline 2038 & & 388 & & 212 & \\
\hline 2039 & & 398 & & 213 & \\
\hline 2040 & & 409 & & 214 & \\
\hline 2041 & & 420 & & 216 & \\
\hline 2042 & & 431 & & 217 & \\
\hline 2043 & & 443 & & 218 & \\
\hline 2044 & & 455 & & 220 & \\
\hline 2045 & & 467 & & 221 & \\
\hline 2046 & & 480 & & 222 & \\
\hline 2047 & & 493 & & 223 & \\
\hline 2048 & & 506 & & 224 & \\
\hline 2049 & & 520 & & 225 & \\
\hline 2050 & & 534 & & 226 & \\
\hline 2051 & & 549 & & 227 & \\
\hline 2052 & & 564 & & 228 & \\
\hline 2053 & & 579 & & 229 & \\
\hline 2054 & & 595 & & 230 & \\
\hline 2055 & & 611 & & 231 & \\
\hline 2056 & & 627 & & 232 & \\
\hline 2057 & & 644 & & 233 & \\
\hline 2058 & & 662 & & 233 & \\
\hline 2059 & & 679 & & 234 & \\
\hline 2060 & & 698 & & 235 & \\
\hline 2061 & & 717 & & 236 & \\
\hline 2062 & & 736 & & 236 & \\
\hline 2063 & & 756 & & 237 & \\
\hline 2064 & & 777 & & 238 & \\
\hline 2065 & & 798 & & 238 & \\
\hline 2066 & & 819 & & 239 & \\
\hline 2067 & & 841 & & 239 & \\
\hline 2068 & & 864 & & 240 & \\
\hline 2069 & & 888 & & 240 & \\
\hline 2070 & & 912 & & 241 & \\
\hline 2071 & & 936 & & 241 & \\
\hline 2072 & & 962 & & 241 & \\
\hline 2073 & & 988 & & 242 & \\
\hline 2074 & & 1014 & & 243 & \\
\hline 2075 & & 1042 & & 243 & \\
\hline 2076 & & 1070 & & 244 & \\
\hline 2077 & & 1099 & & 244 & \\
\hline 2078 & & 1129 & & 245 & \\
\hline 2079 & & 1159 & & 245 & \\
\hline 2080 & & 1191 & & 245 & \\
\hline \multicolumn{2}{|c|}{ Mean Absolute Percentage Error } & & $7.22 \%$ & & $1.18 \%$ \\
\hline
\end{tabular}

We have calculated the predicted population of Bangladesh from the equations (15) and (17), In Table 1 we get that the predicted populations of Bangladesh are expected to be 215 million (approximately) and 171 million (approximately) in 2016 by using Exponential model and the Logistic model respectively.

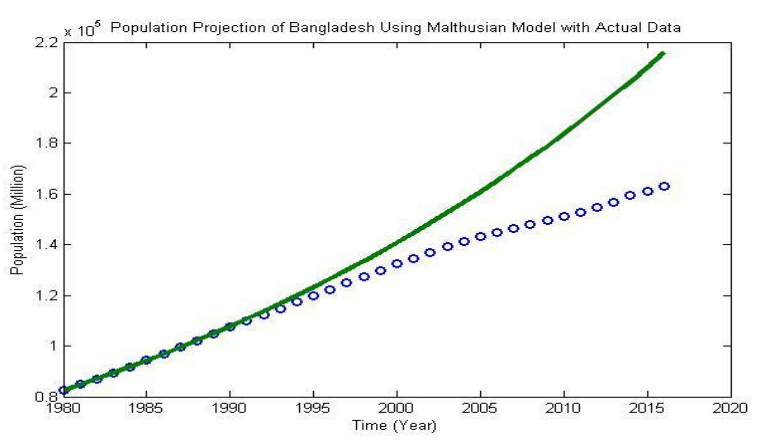

Figure 1. The actual (blue) and predicted (green) populations of Bangladesh using Malthusian model (15) from 1980 to 2016

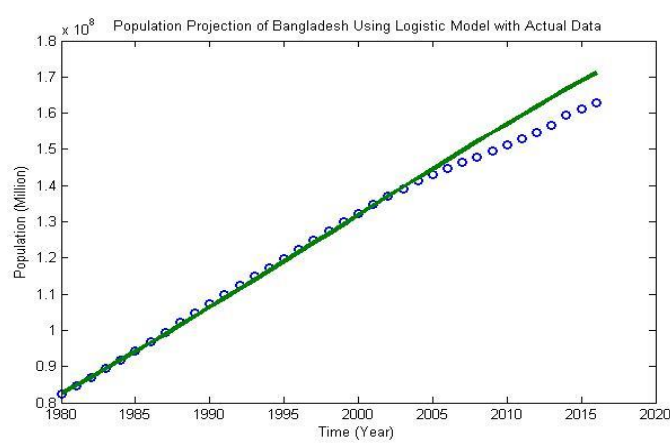

Figure 2. The actual (blue) and predicted (green) populations of Bangladesh using Logistic model (17) from 1980 to 2016

Let us now present the numerical simulation results of the actual and projected populations of Bangladesh from the year 1980 to 2016 using Malthusian model (15) and Logistic model (17) in Figures 1 and 2 respectively.

Now, a combined graph of the Malthusian and Logistic models showing a comparative population size in Bangladesh from 1980 to 2016 is shown in Figure 3. 


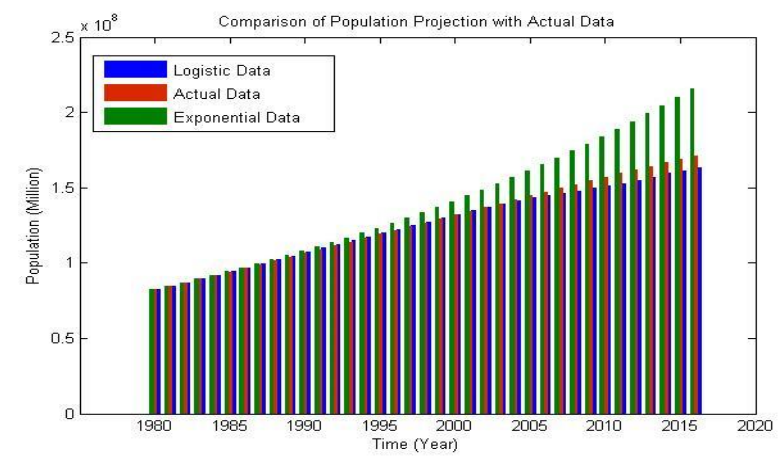

Figure 3. Comparison of population projection between Malthusian and Logistic model with actual data from 1980 to 2016

Again we have calculated the predicted population of Bangladesh from 1980 to 2080 using the equations (15) and (17). As shown in Table 1, we get that the predicted populations of Bangladesh are expected to be 1191 million )approximately( and 245 million )approximately( in 2080 by using Exponential model and the Logistic model respectively. In this situation, population growth is the pressing problem of Bangladesh like every developing country. For our limited resource, it will too difficult to cope with this over population but Bangladesh tries to reduce population growth rate, increase literacy rate and create mass awareness to overcome this problem.

Now we present the numerical simulation results of the actual and projected populations of Bangladesh from the year 1980 to 2080 using Malthusian model (15) and Logistic model (17) in Figures 4 and 5 respectively.

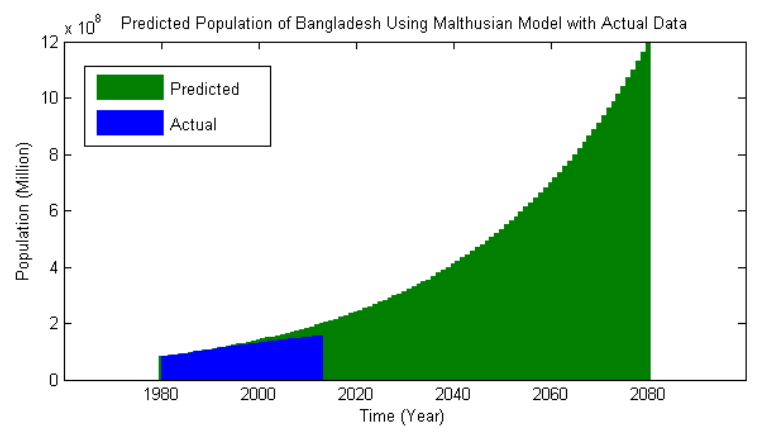

Figure 4. The actual (Blue) and predicted (Green) populations of Bangladesh using Malthusian model (15) from 1980 to 2080

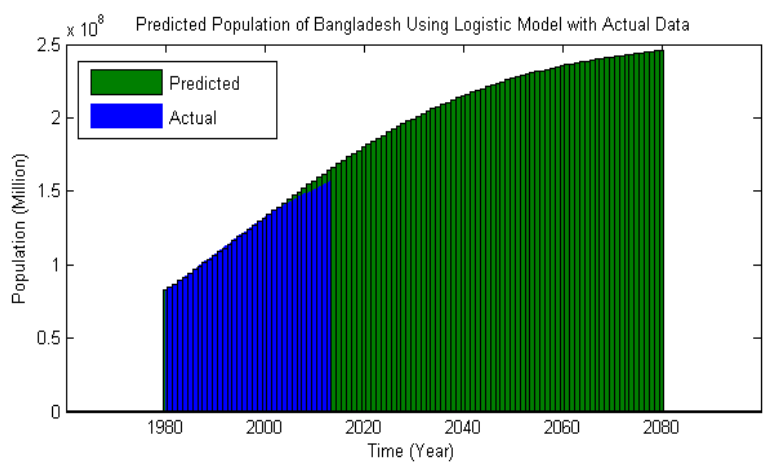

Figure 5. The actual (Blue) and predicted (Green) populations of Bangladesh using Logistic model (17) from 1980 to 2080
Again a combined graph of the Malthusian and Logistic models showing a comparative population size from 1980 to 2080 is shown in Figure 6.

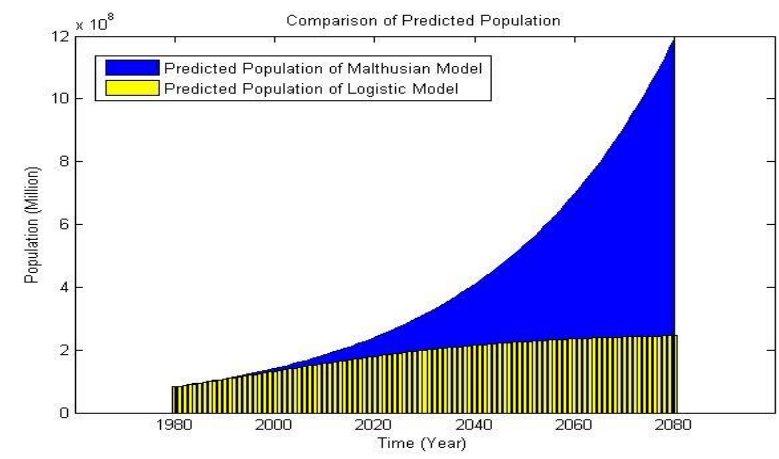

Figure 6. Comparison of population projection between Malthusian and Logistic model from 1980 to 2080

\section{CONCLUSIONS}

In this paper, we consider two simple deterministic population growth models: the exponential and the logistic and make a comparison to predict the future population of Bangladesh. Our analysis gives good approximation for long term prediction of the population growth trends in comparison to present scenario of human population in Bangladesh. We analyze and investigate to predict the populations for 100 years, which gives the carrying capacity of around 245 million and this leads to an estimated population of around 245.76 million in 2080. If we would predict the population for 50 years, the logistic growth yields a straight line showing a good approximation. According to the World Bank data, the population of Bangladesh was around 172.88 million and 195.07 million in the years 2014 and 2015 respectively which are very close to our predicted population in comparison to the same years. Even our predicted population from 1980 to 2016 is almost similar to that of the World Bank data. So, our study provides a better prediction for the future population size of Bangladesh.

Finally, we find that according to the exponential model the predicted growth rate is approximately $2.67 \%$ and predicted population of Bangladesh is 1191 million in 2080 with a Mean Absolute Percentage Error (MAPE) of 7\%. On the other hand, the population growth rate of Bangladesh is approximately $4 \%$ according to logistic model and the carrying capacity for the population of

Bangladesh is calculated as $254.89 \times 10^{6}$. By the Logistic model, the population of Bangladesh is calculated to be 245 million in 2080 with a Mean Absolute Percentage Error (MAPE) of $1.2 \%$. As we know that the vital coefficients are responsible for population growth of any country. So we have calculated the vital coefficients $a$ and $b$ are 0.04 and $1.58 \times 10^{-10}$ respectively. It is also shown that Mean Absolute Percentage Error (MAPE) is very low in logistic model. From this point of view, we can finally conclude that the logistic model gives a good forecasting result as compared to the Malthusian model for a long term prediction.

\section{REFERENCES}

[1] Akçakaya HR, Gulve PS. (2000). Population viability 
analysis in conservation planning: an overview. Ecological Bulletins 48: 9-21. https://doi.org/10.2307/20113245

[2] Biswas MHA, Ara M, Haque MN, Rahman MA. (2011). Application of control theory in the efficient and sustainable forest management. International Journal of Scientific \& Engineering Research 2(3): 26-33.

[3] Biswas MHA, Paiva LT, Pinho MD. (2014). A SEIR model for control of infectious diseases with constraints. Mathematical Biosciences and Engineering 11(4): 761784. https://doi.org/10.3934/mbe.2014.11.761

[4] Biswas HA. (2014). Optimal control of Nipah Virus (NiV) infections: A Bangladesh scenario. Journal of Pure and Applied Mathematics: Advances and Applications 12(1): 77-104.

[5] Biswas HA. (2012). Model and control strategy of the deadly Nipah Virus (NiV) infections in Bangladesh. Research \& Reviews in BioSciences 6(12): 370-377.

[6] Cohen JE. (1995). Population growth and earth's human carrying capacity. American Association for the Advancement of Science 269(5222): 341-346. https://doi.org/10.1126/science.7618100

[7] Deshotel D. (2013). Modeling World Population. Available

http://home2.fvcc.edu/ dhicketh/DiffEqns/spring13proj ects/Population\%20Model\%20Project\%202013/Populat ionModels2013.pdf.

[8] Edwards CH, Penney DE. (2004). Differential equations and boundary value problems computing and modeling. 3rd edition, Pearson Education, Inc.

[9] Farid KS, Ahamed JU, Sharma PK, Begum S. (2011). Population dynamics in Bangladesh: Data sources, current facts and past trends. J Bangladesh Agriculture University $9(1)$ : 121-130. https://doi.org/10.3329/jbau.v9i1.8754

[10] Haque MM, Ahamed F, Anam S, Kabir MR. (2012). Future population projection of Bangladesh by growth rate modeling using logistic population model. Annals of Pure and Applied Mathematics 1(2): 192-202.

[11] Islam MR. (2011). Modeling and predicting cumulative population of Bangladesh. American Journal of Computational and Applied Mathematics 1(2): 98-100.

[12] Islam T, Fiebig DG, Meade N. (2002). Modelling multinational telecommunications demand with limited data. International Journal of Forecasting 18: 605-624. https://doi.org/10.1016/S0169-2070(02)00073-0

[13] Koya PR, Goshu AT. (2013). Generalized mathematical model for biological growths. Open Journal of Modelling and Simulation 1:

$42-53$.

https://doi.org/10.4236/ojmsi.2013.14008

[14] Malthus TR. (1893). An Essay on the Principle of Population. (1st edition, plus excepts 1893 2nd edition), Introduction by Philip Appeman, and assorted commentary on Malthus edited by Appleman, Norton Critical Edition, ISBN 0-393-09202-X.

[15] Mahsin M, Hossain SS. (2012). Population forecasts for Bangladesh, using a Bayesian Methodology. Journal of Health, Population and Nutrition 30(4): 456-463. https://doi.org/10.3329/jhpn.v30i4.13331

[16] Murray JD. (1989). Mathematical Biology. 2nd edition, Springer-Verlag Berlin.

[17] Ofori T, Ephraim L, Nyarko F. (2013). Mathematical modeling of Ghana's population growth. International Journal of Modern Management Sciences 2(2): 57-66.

[18] Pozzi F, Small C, Yetman G. (2002). Modeling the distribution of human population with night-time satellite imagery and gridded population of the world. Proceedings of Pecora 15/Land Satellite Information IV/ISPRS Commission I/FIEOS Conference.

[19] Roy B, Roy SK. (2015). Analysis of prey-predator three species models with vertebral and invertebral predators. International Journal Dynamics and Control 3: 306-312. https://doi.org/10.1007/s40435-015-0153-6

[20] Roy SK, Roy B. (2016). Analysis of prey-predator three species fishery model with harvesting including prey refuge and migration. International Journal of Bifurcation and Chaos 26(1650022). https://doi.org/10.1142/S021812741650022X

[21] Sardar AK, Hanif M, Asaduzzaman M, Biswas MHA. (2016). Mathematical analysis of the two species LotkaVolterra predator-prey inter-specific game theoretic competition model. Advanced Modeling and Optimization 18(2): 231-242.

[22] Tsoularis A, Wallace J. (2001). Analysis of logistic growth models. Res. Lett. Inf. Math. Sci (2): 23-46. https://doi.org/10.1016/S0025-5564(02)00096-2

[23] The World Bank Population Report. Available at http://data.worldbank.org/indicator/SP.POP.TOTL.

[24] Wali A, Ntubabare D, Mboniragira V. (2011). Mathematical modeling of Rwanda's population growth. Applied Mathematical Science 5(53): 2617-2628.

[25] Wali A, Kagoyire E, Icyingeneye P. (2012). Mathematical modeling of Uganda's population growth. Applied Mathematical Science 6(84): 4155-4168. 